\title{
Nafcillin-Induced Allergic Eosinophilic Cholestatic Hepatitis
}

\author{
Mark V. Guido ${ }^{\mathrm{a}}$, Warit Jithpratuck ${ }^{\mathrm{b}}$, Graham E. Parks ${ }^{\mathrm{c}}$, \\ Guha Krishnaswamya, d, e
}

\begin{abstract}
A 71-year-old female with no history of liver disease or antibiotic allergy developed jaundice with elevated liver enzymes and eosinophilia following treatment with nafcillin for septic arthritis. Further workup demonstrated hepatocellular dysfunction and liver biopsy showed expansion of portal tracts by lymphocytes and eosinophils consistent with a hypersensitivity reaction. Nafcillin and related antibiotics were withdrawn, and her symptoms resolved 3 months later. We searched PubMed using terms of "nafcillin cholestasis" and "nafcillin hepatitis", and a review of the literature showed other reports of nafcillin-induced hepatitis and cholestasis. Avoidance and on occasion the guarded use of glucocorticoids can lead to recovery from the insult. This case report shows that while rare, nafcillin can cause cholestatic hepatitis through a likely eosinophil-mediated hypersensitivity reaction. Further studies are needed to elucidate the mechanism of this reaction.
\end{abstract}

Keywords: Allergy; Hepatitis; Cholestasis; Nafcillin; Jaundice

\section{Introduction}

Liver injury associated with the use of various forms of penicillins has been described, with more of a hepatocellular than cholestatic pattern. Often, liver injury is asymptomatic manifesting as abnormalities of routinely monitored liver function tests but severe or even fatal disease may ensue. Nafcillin, a semisynthetic penicillin, has been associated with hepatocellular and/or cholestatic patterns of liver injury. Cholestatic hepatitis has also been described with the oxypeni-

Manuscript submitted March 13, 2017, accepted April 13, 2017

aDepartment of Internal Medicine, Wake Forest Baptist Medical Center, Winston Salem, NC, USA

${ }^{b}$ CJW Medical Center, Richmond, VA, USA

'Department of Pathology, Wake Forest Baptist Medical Center, Winston Salem, NC, USA

dDivision of Allergy and Clinical Immunology, W. G. (Bill) Hefner Veterans Affairs Medical Center, Salisbury, NC, USA

${ }^{\mathrm{e}}$ Corresponding Author: Guha Krishnaswamy, Wake Forest University School of Medicine, Medical Center Boulevard, Watlington Tower, 2nd Floor, Wake Forest Baptist Medical Center, Winston Salem, NC 27157, USA.

Email: gkrishna@wakehealth.edu

doi: https://doi.org/10.14740/gr824w cillins (such as cloxacillin or dicloxacillin), ampicillin and cephalosporins.

In this study, we present a case of nafcillin hypersensitivity manifesting as a cholestatic hepatitis with eosinophil infiltration, summarize the presentation and evolution of nafcillin-induced hepatic injury and discussed oxacillin- and cloxacillin-induced hepatitis. We also review the literature and briefly discuss the mechanisms and clinical implications.

\section{Case Report}

A 71-year-old female presented with oxacillin-sensitive Staphylococcus aureus septic arthritis involving her knee prosthesis. She underwent irrigation and debridement of her left knee with polyethylene exchange, and was discharged on a course of intravenous nafcillin. Towards the end of this 6-week course of nafcillin, the patient presented to the emergency room with anorexia, vomiting and weight loss. She used no other prescription medication other than naproxen for arthritis. The patient was overtly icteric but afebrile. Initial laboratory results were significant for an elevated aspartate transaminase (AST) level of $115 \mathrm{U} / \mathrm{L}$ (normal: 5 - 50), alanine transaminase (ALT) level of $121 \mathrm{U} / \mathrm{L}$ (normal: 5 - 50), total bilirubin $10.9 \mathrm{mg} / \mathrm{dL}$ (normal: < 1.2), direct bilirubin $6.3 \mathrm{mg} / \mathrm{dL}$ (normal: < 0.4 ), and alkaline phosphatase 209 U/L (normal: 20 - 125). Complete blood count on presentation also showed eosinophilia (absolute count 900 cells $/ \mathrm{mm}^{3}$ (normal: 0 - 400), with a total WBC count of 4,300 cells $/ \mathrm{mm}^{3}$ (normal: 4,600 - 10,200). Liver function tests peaked approximately $1-2$ weeks after presentation (Table 1), and these abnormalities returned to normal over 3 months of observation without intervention. Eosinophil counts also climbed and slowly dropped back to normal ranges over months (Fig. 1). Thorough serological, immunological and radiological evaluations failed to demonstrate an etiology. $\operatorname{IgE}$ and $\operatorname{IgG}$ antibodies to penicilloyl G, penicilloyl V, ampicillin and amoxicillin were undetectable. Skin testing with beta-lactam antibiotics was contraindicated by the severity of her underlying illness and hospitalization.

A nuclear medicine hepatobiliary function study showed findings consistent with hepatocellular dysfunction with poor clearance of the radiotracer from the blood pool and no evidence of biliary excretion. A diagnostic liver biopsy was carried out and the histology demonstrated an expansion of portal tracts by lymphocytes, eosinophils, neutrophils and rare plasma cells, edema of portal tracts with ductal reaction, patchy cholestasis and mild microvesicular steatosis (Fig. 2a-d). Nafcillin was discontinued and alternative antibiotics were used 
Table 1. Laboratory Data Observed in the Patient

\begin{tabular}{|c|c|c|}
\hline Laboratory test & Patient (peak value) & Normal \\
\hline White blood cell count (cells $/ \mathrm{mm}^{3}$ ) & 8,200 & $4,600-10,200$ \\
\hline Eosinophils (\%) & 20 & $<6$ \\
\hline Eosinophils (cells $/ \mathrm{mm}^{3}$ ) & 1,640 & 400 \\
\hline Direct bilirubin (mg/dL) & 12.0 & $<0.4$ \\
\hline Total bilirubin (mg/dL) & 22.1 & $<1.2$ \\
\hline Alkaline phosphatase (U/L) & 908 & $20-125$ \\
\hline AST (U/L) & 280 & $5-50$ \\
\hline $\operatorname{ALT}(\mathrm{U} / \mathrm{L})$ & 281 & $5-50$ \\
\hline
\end{tabular}

for her recurrent knee infection.

\section{Discussion}

Nafcillin, a second generation penicillin that is resistant to inactivation by bacterial penicillinase, is commonly used to treat severe staphylococcal infections. Common adverse effects of this drug include gastrointestinal (nausea, vomiting, diarrhea, and dyspepsia), skin eruptions, IgE-mediated hypersensitivity and headache. Hepatocellular injury has been described with nafcillin as well as with oxacillin, cloxacillin and dicloxacillin. Some of the published studies are shown in Table 2 [1-24].

\section{Hepatotoxicity}

Elevations of liver enzymes and evidence of hepatotoxicity have been shown with nafcillin in several studies. In a study by Maraqa and co-workers, adverse drug reactions to oxacillin and nafcillin were evaluated in 222 children receiving parenteral antibiotics in the outpatient setting [25]. Adverse reactions were seen almost twice as common in the oxacillintreated patients compared to those receiving nafcillin. Hepatotoxicity was seen in $3.8 \%$ of the patients, but was strikingly more common in the oxacillin versus the nafcillin group $(22 \%$ versus $0 \% ; \mathrm{P}<0.001)$. In the study reported by Youngster et al, nafcillin was associated with more hepatotoxicity than cefazolin $(8.1 \%$ versus $1.6 \% ; \mathrm{P}<0.01)$ [1]. Of interest, oxacillin-induced transaminitis has also been described, but such patients have been switched to parenteral nafcillin without adverse effects. Hepatotoxic effects with nafcillin have tended to be more associated with intrahepatic cholestasis and rarely with skin eruptions or fever. As in the patient described in this case report, eosinophilia has been described.

\section{Nafcillin-induced cholestasis}

While nafcillin is known to affect bilirubin transport and cause conjugated hyperbilirubinemia, this case report and others suggest that nafcillin can also cause a hypersensitivity-medi-

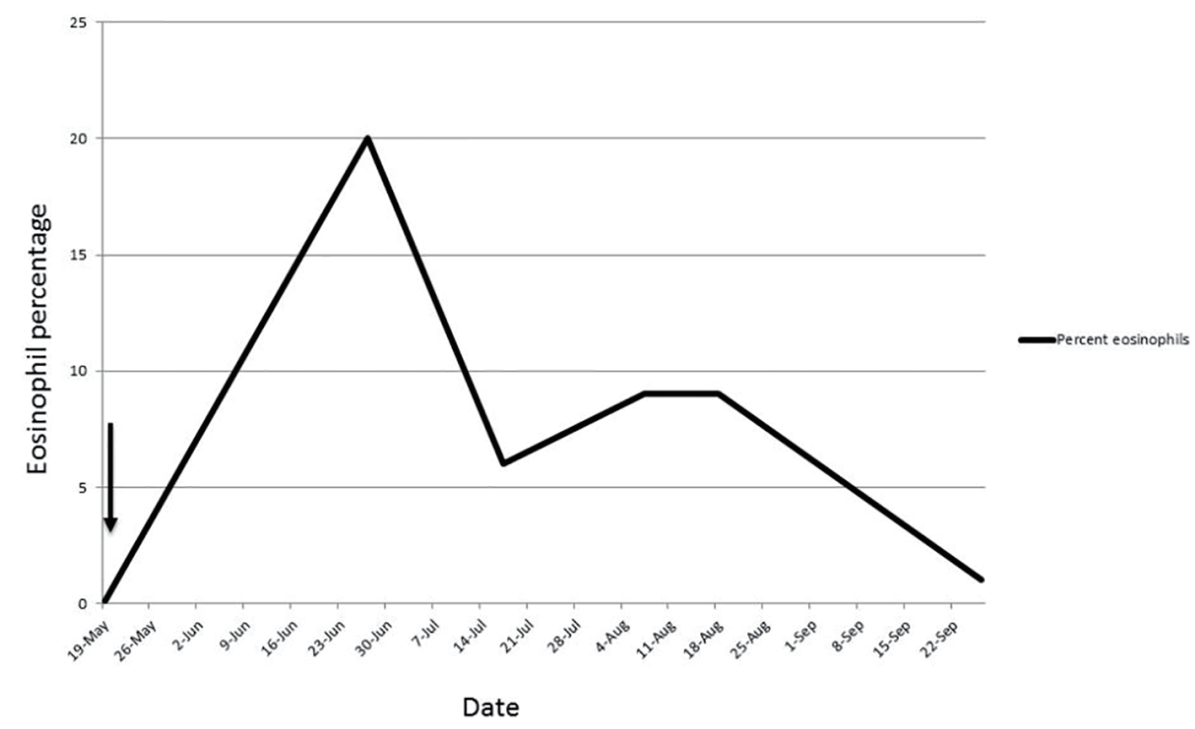

Figure 1. Eosinophilia-course and duration in the patient. Arrow indicates nafcillin start date. 

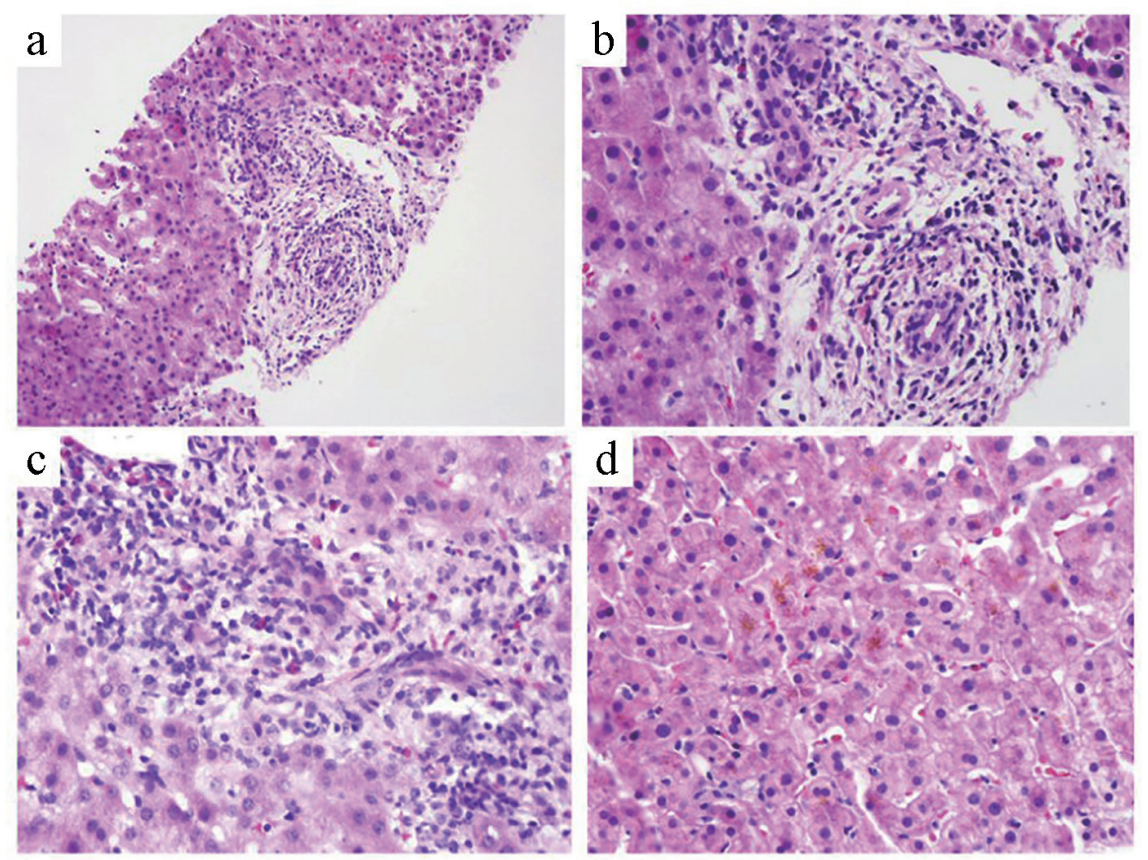

Figure 2. Liver pathology in the patient. Expansion of portal tracts by lymphocytes, eosinophils, rare plasma cells, and bile duct injury are seen $(a: \times 20 ; b: \times 60)$. Focal portal edema with ductular reaction and neutrophilic inflammation is also present (c: $\times$ 40). Patchy cholestasis and mild microvesicular steatosis are observed (d: $\times 40)$.

ated liver injury. The patient described in this study developed an allergic, eosinophilic, cholestatic pattern of liver injury. In one study, 6/46 patients treated with nafcillin developed eosinophilia [2]. In a larger study of 119 patients treated with the antibiotic, $8.1 \%$ of patients developed liver function abnormalities [1]. Nahata and co-workers described transaminitis occurring in one of 32 patients treated with nafcillin [3]. Table 2 lists reported cases of hepatocellular injury induced by various antibiotics and Table 3 [4-7] lists the reported cases of nafcillin-induced hepatocellular disease.

Lestico et al described four cases of hepatic and renal dysfunction following nafcillin therapy [4]. Hepatotoxicity was seen within 3 days of initiating nafcillin with elevated bilirubin peaking at day 5 in most of the cases [4]. The authors commented that risk factors and mechanisms were unclear but

Table 2. Hepatic Injury Reported for Nafcillin and Related Antibiotics [1-24]

\begin{tabular}{lll}
\hline Antibiotic & Hepatic injury pattern & References \\
\hline Nafcillin & $\begin{array}{l}\text { Predominantly cholestasis } \\
\text { Eosinophilia }\end{array}$ & {$[1-7]$} \\
& Hypersensitivity & \\
Oxacillin & $\begin{array}{l}\text { Hepatitis } \\
\text { Rare cholestasis } \\
\text { Transaminitis } \\
\text { Eosinophilia in majority }\end{array}$ & \\
& Hypersensitivity & \\
& Cholestatic hepatitis & {$[19-24]$} \\
Cloxacillin & & \\
\hline
\end{tabular}

in many cases, renal dysfunction was also observed. Presti et al discussed the propensity for nafcillin to induce prolonged cholestatic hepatitis with a delayed resolution, as happened in our patient [5]. In their report, the authors described the use of nafcillin for lower extremity cellulitis in a 63-year-old woman with rheumatoid arthritis. The patient had been treated with glucocorticoids and methotrexate for 2 years prior to the development of cellulitis. On the ninth day of hospitalization, 5 days after starting nafcillin, the patient developed icterus which was associated with elevations of serum bilirubin, transaminases and alkaline phosphatase. Liver biopsy demonstrated findings consistent with an inflammatory cholestasis and infiltration of portal triads with lymphocytes and eosinophils. Macrovesicular steatosis and intracellular and canalicular cholestasis were also observed. Cholestasis and laboratory abnormalities persisted for over 40 - 70 days after initiation of nafcillin.

Mazuryk and co-workers described cholestatic hepatitis following nafcillin administration that responded to low dose prednisone therapy [6]. The patient, an 80-year-old woman with a history of palindromic rheumatism was treated with nafcillin for septic arthritis of her left knee. The patient developed skin eruption, pruritus and diarrhea 2 weeks into nafcillin therapy. Nafcillin was discontinued but the patient continued to have progressive hepatocellular dysfunction and cholestasis with elevated bilirubin and alkaline phosphatase. Liver biopsy demonstrated centrilobular cholestasis and periportal infiltration by mononuclear cells and eosinophils [6]. Prednisone was initiated with a prompt decrease in bilirubin and alkaline phosphatase levels and improvement in pruritus. A repeat liver biopsy demonstrated resolution of inflammation and prednisone was tapered slowly over 26 days. 
Table 3. Salient Features of the Reported Cases of Nafcillin-Induced Hepatic Injury [4-7]

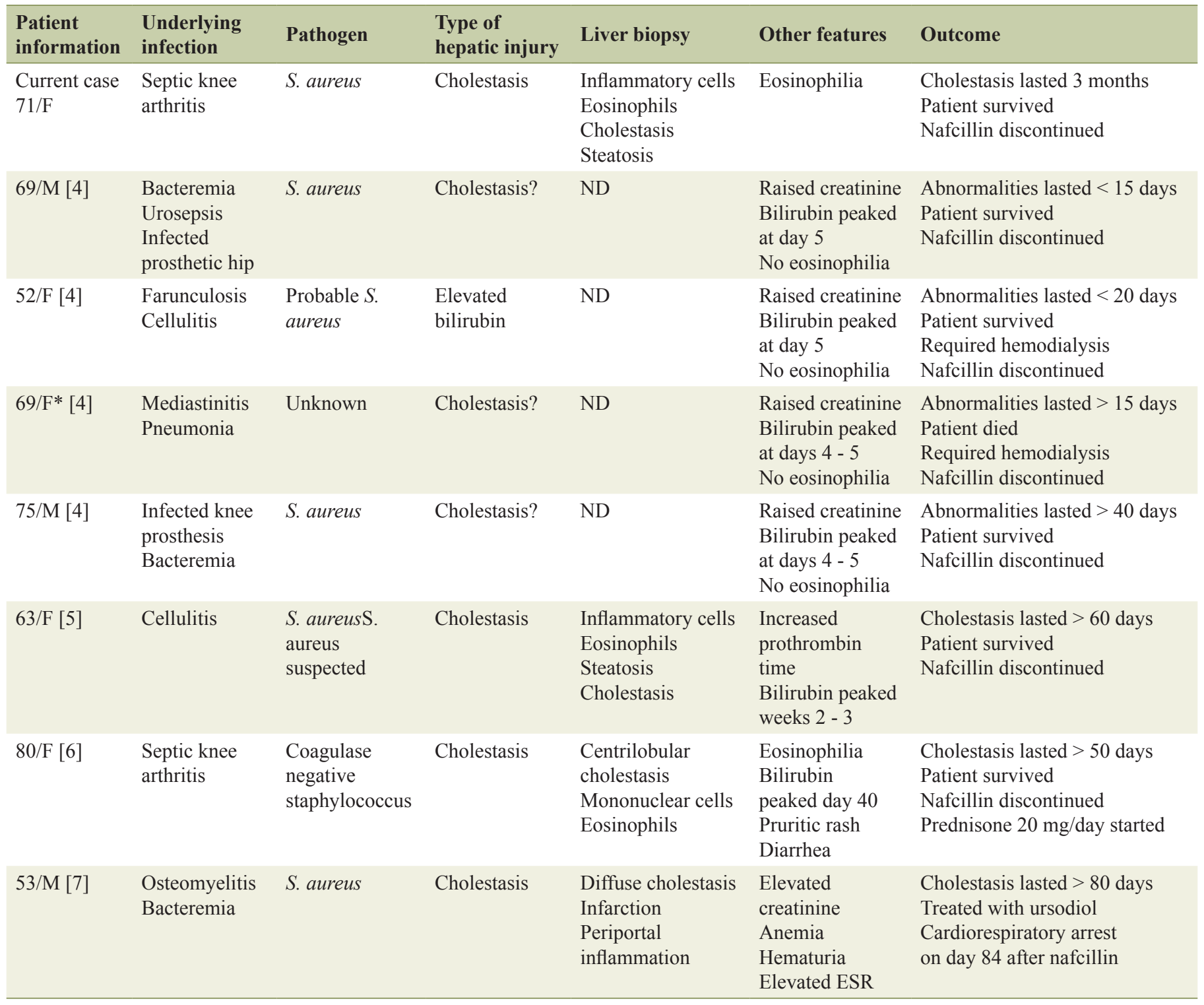

Nafcillin-induced fatal hepatotoxicity associated with very elevated bilirubin levels has also been described [7]. Alam and co-workers reported that a 53-year-old woman with prior history of diabetes and heart disease developed severe transaminitis and cholestasis starting day 15 after starting nafcillin for staphylococcal bacteremia and osteomyelitis of the toe [7]. Nafcillin was subsequently discontinued immediately on day 15 after recognition of hepatic injury; however, this patient finally succumbed to cardiorespiratory arrest 84 days after nafcillin was initiated.

As shown in Table 3, ages ranged between 52 and 80 years, with a dominance in female patients. Adverse effects of nafcillin included septic arthritis, cellulitis, mediastinitis, urosepsis and osteomyelitis. In four cases, positive Staphylococcus aureus cultured was identified from tissue to blood, while in one patient, the pathogen was coagulase negative Staphylo- coccus aureus. The dominant pathology was cholestasis, with rash and arthralgia in one patient, eosinophilia in two patients and renal failure in five. The duration of cholestatic hepatitis varied between 15 days and 3 months or more. Two patients died and one patient was treated with prednisone that allowed rapid recovery of liver function.

\section{Oxacillin/dicloxacillin reactions and hepatotoxicity}

Several reports in the last few decades have reported on the hepatotoxic potential of oxacillin and related antibiotics [816, 26]. The adverse effect has been seen in both children and adults and reports have demonstrated various degrees of severity. Bruckstein and Attia reported on two patients with oxacillin hepatitis [8]. The study reported on transient hepatitis in two 
patients with staphylococcal endocarditis treated with oxacillin intravenously. Elevated transaminases were observed along with non-specific hepatitis on biopsy. The transaminitis resolved when the antibiotic was discontinued suggesting this to be a reversible phenomenon. The authors reviewed other nine reported cases of oxacillin-induced hepatitis, with seven occurring in children 16 years and younger [8]. In their discussion, the authors conclude that this was likely a "hypersensitivity phenomenon" rather than a toxic side effect but the absence of a skin eruption, arthralgia and eosinophilia suggested that these events were not true "allergic" responses. In most cases, the adverse effect was subclinical and discovered by monitoring liver enzymes. Olans and co-workers also described eight patients with reversible oxacillin-induced hepatocellular injury which resulted in asymptomatic transaminitis [17]. Patients with oxacillininduced transaminitis have been switched to nafcillin without adverse consequences. Flucloxacillin and cloxacillin have also been associated with a form of cholestatic hepatitis [18-23].

\section{Mechanisms}

While other penicillins such as penicillin $\mathrm{G}$ and methicillin are cleared by the kidneys, nafcillin is hepatically excreted and becomes highly concentrated in bile [24]. In patients with pre-existing liver disease, nafcillin elimination half-life is prolonged leading to the speculation that drug-induced hepato-toxicity might further impair nafcillin clearance and lead to more liver injury. Other studies have also shown that nafcillin leads to decreased bilirubin excretion and bilirubin concentration in bile leading to a hyperbilirubinemia [27]. These effects may be mediated by influencing transport of bilirubin conjugates from hepatocytes across canalicular membranes into biliary fluid and such competition for transport pathways can lead to hyperbilirubinemia, at least in a murine model. It is likely that such a common transport system exists for most monobasic beta-lactam antibiotics such as nafcillin and cloxacillin, explaining the propensity of these drugs to cause cholestasis [28]. In some patients receiving the drug, probable genetic predisposition may also allow hepatic immune responses leading to idiosyncratic adverse drug reactions culminating in biliary ductal injury, inflammation and eosinophilia. In some such cases, eosinophilia and/or eosinophil infiltration of hepatic tissue is seen, suggesting an immune-allergic mechanism, probably mediated by mast cells [29]. HLA associations have not been described with nafcillin although a link to HLA-B*57:01-based $\mathrm{T}$ lymphocyte restriction has been reported with flucloxacillininduced liver disease [30]. Due to the rarity of these cases, the exact mechanism has not been evaluated and requires more study in affected patients.

\section{Conclusions}

In summary, a serious but often unrecognized complication of prolonged high dose nafcillin therapy is immunoallergic cholestasis and hepatocellular dysfunction which could be insidious upon onset. Careful monitoring of liver functions and stopping the drug at the first sign of liver function test abnor- malities might prevent progression to serious or fatal liver disease. Such complications could be treated with glucocorticoids if an allergic mechanism is discerned and no contraindications to the use of glucocorticoids exist.

\section{References}

1. Youngster I, Shenoy ES, Hooper DC, Nelson SB. Comparative evaluation of the tolerability of cefazolin and nafcillin for treatment of methicillin-susceptible Staphylococcus aureus infections in the outpatient setting. Clin Infect Dis. 2014;59(3):369-375.

2. Feldman WE, Nelson JD, Stanberry LR. Clinical and pharmacokinetic evaluation of nafcillin in infants and children. J Pediatr. 1978;93(6):1029-1033.

3. Nahata MC, DeBolt SL, Powell DA. Adverse effects of methicillin, nafcillin and oxacillin in pediatric patients. Dev Pharmacol Ther. 1982;4(3-4):117-123.

4. Lestico MR, Vick KE, Hetsko CM. Hepatic and renal dysfunction following nafcillin administration. Ann Pharmacother. 1992;26(7-8):985-990.

5. Presti ME, Janney CG, Neuschwander-Tetri BA. Nafcillin-associated hepatotoxicity. Report of a case and review of the literature. Dig Dis Sci. 1996;41(1):180-184.

6. Mazuryk H, Kastenberg D, Rubin R, Munoz SJ. Cholestatic hepatitis associated with the use of nafcillin. Am J Gastroenterol. 1993;88(11):1960-1962.

7. Alam MB, Kadoura A, Sathaiah M. A fatal case of nafcillin-induced hepatotoxicity: a case report and the literature review. Case Rep Med. 2012;2012:953714.

8. Bruckstein AH, Attia AA. Oxacillin hepatitis. Two patients with liver biopsy, and review of the literature. Am J Med. 1978;64(3):519-522.

9. Halloran TJ, Clague MD. Hepatitis associated with highdose oxacillin therapy. Arch Intern Med. 1979;139(3):376377.

10. Klein I, Tobias H. Oxacillin-associated hepatitis. Am J Gastroenterol. 1976;65(6):546.

11. Lee CY, Chen PY, Huang FL, Chi CS. Reversible oxacillin-associated hepatitis in a 9-month-old boy. J Paediatr Child Health. 2008;44(3):146-148.

12. Onorato IM, Axelrod JL. Hepatitis from intravenous high-dose oxacillin therapy: findings in an adult inpatient population. Ann Intern Med. 1978;89(4):497-500.

13. Pas AT, Quinn EL. Cholestatic Hepatitis Following the Administration of Sodium Oxacillin. JAMA. 1965;191:674-675.

14. Pollock AA, Berger SA, Simberkoff MS, Rahal JJ, Jr. Hepatitis associated with high-dose oxacillin therapy. Arch Intern Med. 1978;138(6):915-917.

15. Taylor C, Corrigan K, Steen S, Craig C. Oxacillin and hepatitis. Ann Intern Med. 1979;90(5):857-858.

16. Trevenzoli M, Cattelan AM, Mencarelli R, Meneghetti F. Severe hepatitis associated with oxacillin therapy. South Med J. 2003;96(3):324-325.

17. Olans RN, Weiner LB. Reversible oxacillin hepatotoxicity. J Pediatr. 1976;89(5):835-838.

18. Goland S, Malnick SD, Gratz R, Feldberg E, Geltner D, 
Sthoeger ZM. Severe cholestatic hepatitis following cloxacillin treatment. Postgrad Med J. 1998;74(867):59-60.

19. Devereaux BM, Crawford DH, Purcell P, Powell LW, Roeser HP. Flucloxacillin associated cholestatic hepatitis. An Australian and Swedish epidemic? Eur J Clin Pharmacol. 1995;49(1-2):81-85.

20. Derby LE, Jick H, Henry DA, Dean AD. Cholestatic hepatitis associated with flucloxacillin. Med J Aust. 1993;158(9):596-600.

21. Miros M, Kerlin P, Walker N, Harris O. Flucloxacillin induced delayed cholestatic hepatitis. Aust N Z J Med. 1990;20(3):251-253.

22. Pascual J, Orofino L, Marcen R, Quereda C, Ortuno J. Cloxacillin-induced cholestasis in a renal allograft patient with chronic hepatitis. Am J Gastroenterol. 1990;85(3):335-336.

23. Kleinman MS, Presberg JE. Cholestatic hepatitis after dicloxacillin-sodium therapy. J Clin Gastroenterol. 1986;8(1):77-78.

24. Marshall JP, 2nd, Salt WB, Elam RO, Wilkinson GR, Schenker S. Disposition of nafcillin in patients with cirrhosis and extrahepatic biliary obstruction. Gastroenterol- ogy. 1977;73(6):1388-1392.

25. Maraqa NF, Gomez MM, Rathore MH, Alvarez AM. Higher occurrence of hepatotoxicity and rash in patients treated with oxacillin, compared with those treated with nafcillin and other commonly used antimicrobials. Clin Infect Dis. 2002;34(1):50-54.

26. Al-Homaidhi H, Abdel-Haq NM, El-Baba M, Asmar BI. Severe hepatitis associated with oxacillin therapy. South Med J. 2002;95(6):650-652.

27. Eng C, Javitt NB. Effect of nafcillin on hepatic excretory function. Biochem Pharmacol. 1983;32(23):3649-3651.

28. Tamai I, Terasaki T, Tsuji A. Evidence for the existence of a common transport system of beta-lactam antibiotics in isolated rat hepatocytes. J Antibiot (Tokyo). 1985;38(12):1774-1780.

29. Shakoory B, Fitzgerald SM, Lee SA, Chi DS, Krishnaswamy G. The role of human mast cell-derived cytokines in eosinophil biology. J Interferon Cytokine Res. 2004;24(5):271-281.

30. Usui T, Naisbitt DJ. Human leukocyte antigen and idiosyncratic adverse drug reactions. Drug Metab Pharmacokinet. 2017;32(1):21-30. 